Cite this: Phys. Chem. Chem. Phys., 2013, 15, 3320

Received 22nd November 2012, Accepted 16th January 2013

DOI: $10.1039 / \mathrm{c} 3 \mathrm{cp} 44163 f$

www.rsc.org/pccp

\title{
Adsorbed and near surface structure of ionic liquids at a solid interface $\dagger$
}

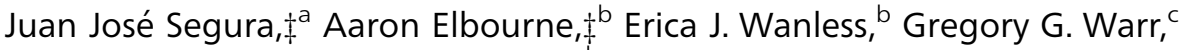 \\ Kislon Voïtchovsky*a and Rob Atkin ${ }^{* b}$
}

\begin{abstract}
The structure of solid-ionic liquid (IL) interfaces has been characterised with unprecedented clarity by employing a range of atomic force microscopy (AFM) imaging techniques and tip pressures appropriate for the system under study. Soft contact and amplitude-modulation (AM) AFM imaging have been used to elucidate the lateral structure of ILs adsorbed onto mica, and in the near surface ion layers. Data is presented for ethylammonium nitrate (EAN) and 1-ethyl-3-methylimidazolium bis(trifluoro-methylsulfonyl)imide (EMIm TFSI). Whereas EAN is a protic IL that forms a nanostructured sponge phase in the bulk, EMIm TFSI is aprotic and has weak (or absent) bulk association structure. Comparison of results obtained for the two liquids elucidates how the strength of bulk liquid morphology effects lateral organisation at the surface, and any effect of IL class, i.e. protic versus aprotic. Imaging reveals EAN self assembles at the solid surface in a worm-like morphology, whereas EMIm cations adsorb in a more isolated fashion, but still in rows templated by the mica surface. To the authors' knowledge, the wormlike structures present at the EAN-mica interface are the smallest self-assembled aggregates ever imaged on a solid surface.
\end{abstract}

\section{Introduction}

ILs are pure salts with melting points less than $100{ }^{\circ} \mathrm{C}$. They are attracting intense research interest on account of their "tuneability", 5,6 which refers to the ability to predictably change physical properties through systematic variation in the structure of the cations and anions, their often negligible vapour pressures and wide temperature and (frequently) redox stability ranges. Most IL cations contain a quaternary nitrogen or phosphorus group attached to an alkyl chain, and are thus surfactant-like ${ }^{2}$ in that they consist of charged and apolar regions. This means that many IL cations exhibit 'solvophobic'7 alkyl chain clustering into apolar domains; analogous to the hydrophobic effect ${ }^{8}$ in water. Many ILs are thus heterogeneous, ${ }^{3,9,10}$ with charged and apolar domains in the bulk liquid forming a bicontinuous nanostructure analogous to a microemulsion or sponge phase, but with length scales around an order of magnitude smaller. In a further analogy to aqueous

\footnotetext{
${ }^{a}$ Department of Materials Science and Engineering, Ecole Polytechnique Fédérale de Lausanne, 1015 Lausanne, Switzerland. E-mail: kislon.voitchovsky@epfl.ch

${ }^{b}$ Discipline of Chemistry, The University of Newcastle, NSW 2308, Callaghan, Australia. E-mail: rob.atkin@newcastle.edu.au

${ }^{c}$ School of Chemistry, The University of Sydney, NSW 2006, Australia

$\dagger$ Electronic supplementary information (ESI) available. See DOI: 10.1039/ c3cp44163f

‡ These two authors contributed equally.
}

surfactant structures, ${ }^{11}$ the IL sponge develops into layers in the vicinity of macroscopic solid surfaces. ${ }^{12-14}$

Together with our collaborators, we have developed a model for describing IL interfacial structure ${ }^{13,15-17}$ which consists of three regions. The interfacial innermost layer comprises the layer of ions in direct contact with the surface of the other phase. This layer is often well organised, and enriched in one ion or the other depending on the nature of the second material. The transition zone is the region over which the strong interfacial layer structure decays to the bulk morphology. The thickness of this zone reflects the spatial rate of change between interfacial and bulk structure. The third zone is the bulk liquid, which frequently has a bicontinuous structure, but can be more homogeneous for short alkyl chain aprotic ILs. For the purposes of these definitions it does not matter whether self assembly occurs in the bulk liquid; the key concept is that interactions between the surface and the IL lead to enrichment of one ion species at the interface, which propagates an anisotropic interfacial structure that decays through the transition zone to the bulk morphology.

This model was developed using data obtained from atomic force microscopy (AFM) ${ }^{6,16}$ X-ray reflectivity, ${ }^{18}$ and vibrational sum frequency spectroscopy (VSFS), ${ }^{19,20}$ amongst others. ${ }^{21,22}$ Aside from VSFS, which probes the orientation of non-centrosymmetric ions in the interfacial layer, these techniques probe the composition of the liquid normal to the interface, and the details of the 
model reflect this; no information about the organisation of the ions in the plane of the interface is provided, aside from the inference that ion layers become less well-defined with distance from the interface.

Here we present new results which reveal the lateral molecular organisation of the IL interfacial structure. Our results combine two complementary AFM-based approaches, both operated with the probing tip/cantilever fully immersed into the ionic liquid: soft contact AFM and amplitude modulation AFM. In soft contact AFM the tip scans using a constant force. In AM-AFM, the base of the cantilever is oscillated while the tip sample-distance is continuously adjusted so that the tip vibration amplitude is constant during scanning. In both modes, topographic images are derived from corrections imposed by a feedback loop to maintain a constant scanning parameter (force or amplitude). In AM-AFM additional information regarding the IL's local viscoelastic properties is derived from the phase lag between the driving vibration at the base of the cantilever and the vibration at the tip. Critically, the AFM tip is able to displace the EMIm cation from the surface and move into contact with the mica substrate, whereas ethylammonium remains adsorbed to mica even at high imaging force, and the lateral cohesion within layers is high. These results will impact upon a wide range of IL fields where surface-IL interactions play a role, including modelling of the IL electrical double layer and colloid stability in ILs, ${ }^{23-28}$ charge storage devices, ${ }^{29,30}$ electrodeposition, ${ }^{31}$ dye solar cells, ${ }^{32}$ and lubrication, ${ }^{33-35}$ amongst others.

\section{Experimental section}

EAN was sythesised via slow addition of concentrated nitric acid $\mathrm{HNO}_{3}$ (AJAX Finechem pty. ltd., $70 \mathrm{w} / \mathrm{w} \%$ ) to a chilled solution $\left(<10{ }^{\circ} \mathrm{C}\right)$ of hydrogenous ethylamine (Aldrich $\left.68 \mathrm{wt} \%\right)$ and $\mathrm{RO}$ water. During the acid addition, the mixture was rapidly stirred to ensure dispersal of any heat generated. Firstly, the sample was rotary evaporated for several hours at $40{ }^{\circ} \mathrm{C}$. The resultant solution was heated overnight in an oil bath at $105{ }^{\circ} \mathrm{C}$ under a nitrogen atmosphere. The liquid was thoroughly purged with filtered nitrogen gas between rotor evaporation and oil bath steps to prevent the formation of nitrous oxide impurities. The water content of these ILs was undetectable by Karl Fisher titration prior to experimentation $(<0.01 \mathrm{v} / \mathrm{v} \%)$. Mica (Brown Co., Sydney) was prepared by using adhesive tape to cleave along the basal plane.

EMIm TFSI (1-ethyl-3-methylimidazolium bis(trifluoromethane sulfonyl)imide) $>99 \%$ pure (IoLiTec, Heilbronn, Germany) was used for the experiments with an aprotic IL. All experiments with EMIm TFSI were carried out in ambient conditions. Since EMIm TFSI is a hydrophobic IL, no special measures were taken to avoid water contamination.

At Newcastle, the EAN-mica interface was studied using a Asylum Research Cypher Atomic Force Microscope (Cypher AFM). Data was obtained at a constant temperature of $25{ }^{\circ} \mathrm{C}$. Force curves were recorded in contact mode and AFM images were obtained in soft contact ${ }^{1}$ mode to minimize deformation of the surface structure. $\mathrm{Si}_{3} \mathrm{~N}_{4}$ tips were used for all experiments (nominal spring constant $k_{\mathrm{c}}=0.06 \mathrm{~N} \mathrm{~m}^{-1}$ ) and were irradiated with UV light for 15 minutes prior to experiment. Scan sizes for force curves were between $30 \mathrm{~nm}$ and $50 \mathrm{~nm}$. The environmental cell for the Cypher AFM is still being developed, so experiments were completed in a droplet exposed to the atmosphere within the AFM box (a sealed enclosure). As EAN is hygroscopic, the water content of the liquid will increase over the course of an experiment. The water content of EAN was routinely checked after an experiment. Even after 4 hours the water content never exceeded $3 \mathrm{wt} \%$. The force curve and images presented in this paper were obtained within no more than 30 minutes of the EAN droplet being placed on the mica. Karl Fischer titration of EAN collected from the cell after this time period had a value of $\sim 1 \mathrm{wt} \%$ that depended slightly on the ambient humidity, so the water concentration in the data presented will be less than this value.

At Lausanne, investigations of the EMIm TFSI-mica interface were completed using two different instruments: a Cypher (Asylum Research, Santa Barbara, USA) and a Multimode Nanoscope IIIA (Bruker, Madison, USA) with a dedicated liquid cell on each system. Prior to each experiment, the cells were thoroughly cleaned by subsequent wiping with acetone, acetonitrile, ethanol using ultraclean photography lens paper (Cypher) and sonication in milli-Q water, isopropanol and milli-Q water followed by drying under nitrogen (Multimode). Experiments completed using the multimode used a closed liquid cell that prevents water ingress. Experiments completed using the Cypher AFM were open to the atmosphere, as per the EAN experiments at Newcastle. However, EMIm is a hydrophobic IL, so water contents will be much lower than those measured for EAN.

The mica substrates consisted of sheet discs (grade 4, SPI supplies, West Chester, USA) freshly cleaved using adhesive tape just before performing each experiment. A drop $(50 \mu \mathrm{L})$ of EMIm TFSI was then deposited on top of the mica substrate and the AFM tip lowered into the IL for imaging.

Imaging in AM-AFM was carried out with the levers oscillated close to resonance with a typical free oscillation amplitude of $A_{0} \sim 1 \mathrm{~nm} .{ }^{36}$ Cantilevers exhibiting different stiffness were used in order explore the whole mica-EMIm TFSI interface layer by layer. The softer levers (OMCL-RC800-PSA and BL-AC40TS-C2, Olympus, Japan, nominal spring constant $k_{\mathrm{c}}=0.76 \mathrm{~N} \mathrm{~m}^{-1}$ and $k_{\mathrm{c}}=0.09 \mathrm{~N} \mathrm{~m}^{-1}$ respectively) were used to image the superficial layers of the interface, further away from the mica substrate (outer interface). A stiffer lever (Arrow UHFAuD NanoWorld, Switzerland, nominal spring constant $k_{\mathrm{c}}=6 \mathrm{~N} \mathrm{~m}^{-1}$ ) was employed to image the EMIm TFSI layers closer to the mica (inner interface). Each cantilever was calibrated using its thermal spectrum prior to imaging and the lever sensitivity determined using force spectroscopy.

For a given cantilever, the phase signal (lag between the driving and the detected oscillations) consistently decreased as the vibrating tip approached the surface. The phase information was therefore used as an indicator of the effective tip-mica distance within the interface (see ESI $\dagger$ for details).

The free oscillation amplitude $A_{0}$ of the tip was always set larger than the thickness of a typical IL layer so as to allow for stable imaging. When working with a given cantilever, the region of the interface explored (inner or transition) was controlled by 
adjusting the imaging amplitude (setpoint). In this paper, we expressed the setpoint as the ratio of $A / A_{0}$. Soft setpoints $\left(A / A_{0} \sim 0.8\right)$ were used for imaging the more delicate outer interfacial layers while harsher setpoints $\left(A / A_{0}<0.6\right)$ gave access to the inner IL layer closer to mica. All images were acquired at typical rates of 1-5 Hz.

\section{Results and discussion}

A force-distance curve for the approach of a silicon nitride AFM tip immersed in EAN towards the mica substrate is presented in Fig. 1(a). These data are in accordance with that described previously. ${ }^{12,37,38}$ The van der Waals and electrical double layer forces that dominate in aqueous systems are electromagnetic in origin, so are effectively screened by the $11 \mathrm{M}$ ionic strength of EAN. The measured force profile is thus primarily due to the IL structure confined between the tip and the surface, and consists of a series of steps that are a consequence of transition zone layers. ${ }^{12}$ The force required to rupture a layer increases with decreasing separation, reflecting greater order near the surface. At large separations (in this case $>2 \mathrm{~nm}$ ) no force is measured as the probe moves through the bulk liquid towards the substrate. A weak step can just be discerned from the data noise at $2 \mathrm{~nm}$, which resists approach until a force of $\sim 1 \mathrm{nN}$ is reached and the layer is ruptured. The tip jumps inward by $0.55 \mathrm{~nm}$ to the next solvent layer. This jump distance is consistent with the molecular dimensions of an EAN ion pair ${ }^{39}$ and is half of the Bragg peak spacing determined from small
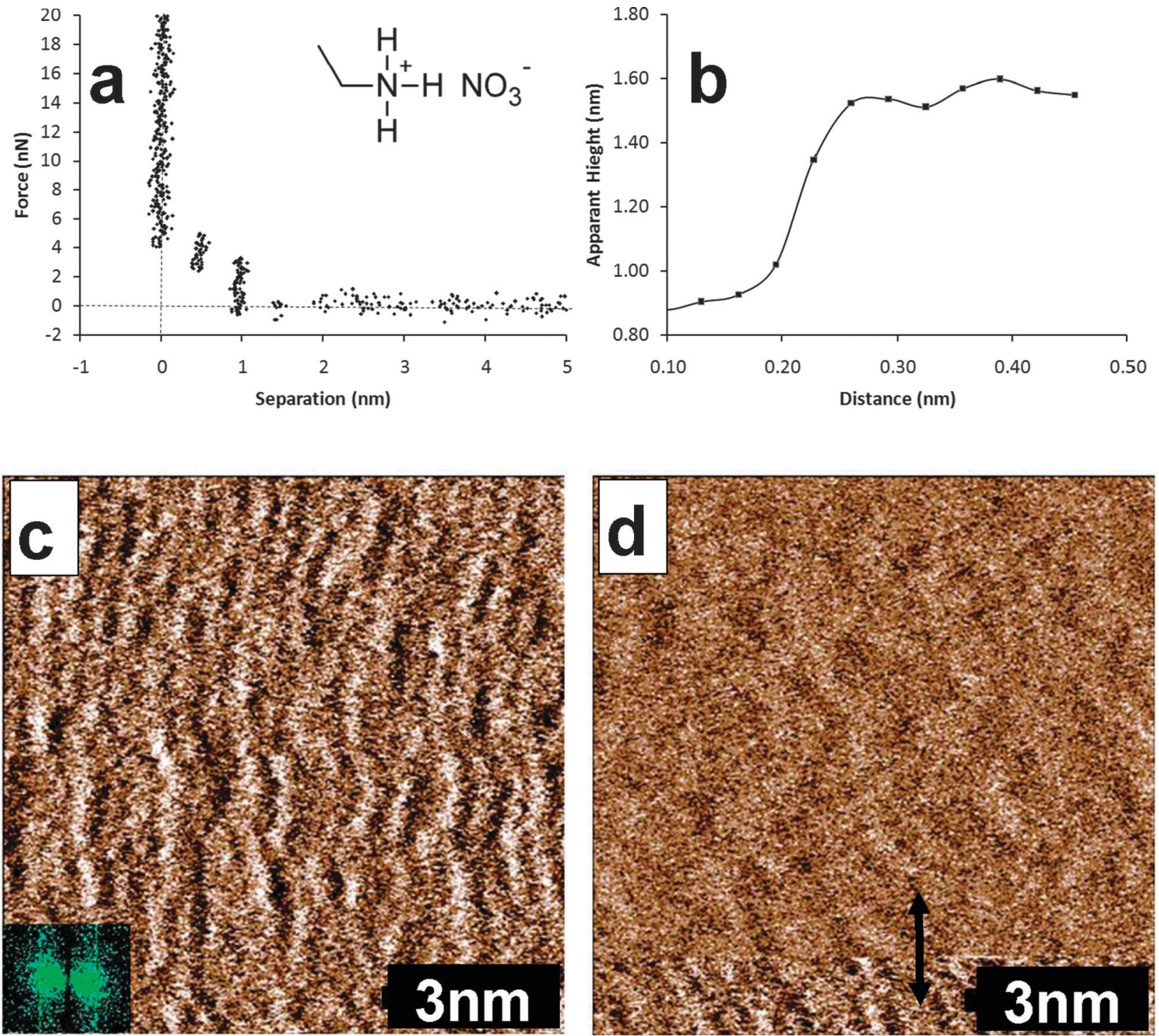

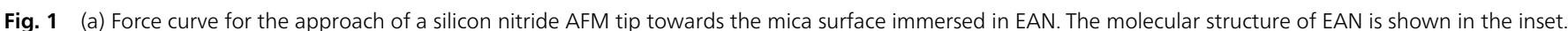

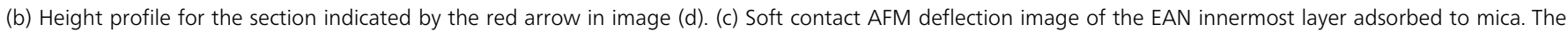

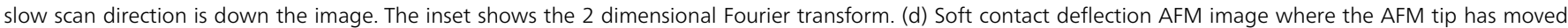

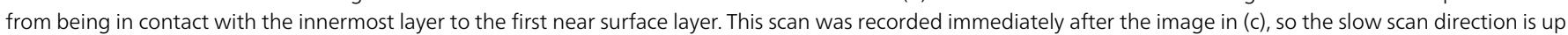
the image. 
angle neutron scattering. ${ }^{2}$ The process of pushing against and rupturing layers is repeated as the tip makes its way towards the surface and an apparent separation of zero is reached. The term apparent separation is used because the zero position in an AFM force curve is determined from the compliance region in the raw data, and the true separation is unknown. In previous articles, ${ }^{12,14,34}$ we have argued that for the EAN mica system the zero position corresponds to the innermost layer of strongly adsorbed cations that cannot be displaced by the AFM tip, even at high force. The AFM images presented in this work substantiate this interpretation, but also that the cations in the surface bound layer self assemble, which was not previously suspected.

A soft contact image of the innermost interfacial layer for the EAN-mica system is presented in Fig. 1(c). The slow scan direction is down the image. This image was obtained in soft contact mode at a force slightly higher than that required to push through the final EAN transition zone layer, to minimize the risk of the tip inducing structure in the innermost layer. Images obtained at much higher force, described below, markedly change the features, which provides confidence that this image is a true representation of the unperturbed state. Fig. 1(c) reveals slightly disordered worm-like structures running perpendicular to the scan direction. These structures rotated as the scan direction was changed, and their size scaled correctly as the image size was increased and decreased, confirming that they are not imaging artefacts. The inter-aggregate periodicity was determined from the two-dimensional Fourier transform to be $5 \pm 0.3 \AA$ independent of scan angle, which is identical within error to the mica lattice spacing determined by the same method in air (image not shown).

Fig. 1(d) shows a typical image obtained when the force applied to the AFM tip is allowed to drift below the penultimate push-through force during the scan, such that the tip moves out of contact with the surface bound ions and images the first near surface layer. This image was recorded immediately after Fig. 1(c) so the scan direction is up the image. In the bottom portion of the image, the tip is in contact with the surface bound ions, and the structure has the same appearance as that shown in Fig. 1(c). About $1 \mathrm{~nm}$ from the bottom of the image, the appearance changes markedly. The section analysis shown in Fig. 1(b) reveals that the height difference that accompanies the structure change is $\sim 0.5 \mathrm{~nm}$, consistent with the step width of the force curve in Fig. 1(a), and of an EAN ion pair, confirming the tip has jumped from the interfacial to the first near-surface layer. The lateral structure of the first near-surface or transition zone layer is less well defined than the innermost interfacial layer, but is also consistent with a 2 dimensional representation of a sponge structure. ${ }^{3,9,40}$ The features are larger, and the depth difference between high and low points in the height image (see ESI, $\uparrow$ Fig. A and B) is smaller than in the interfacial layer, consistent with a level of organization intermediate between the interfacial and bulk morphologies.

Bulk liquid EAN has a sponge structure that can be conceptualised at the molecular level as a disordered bilayer. A freshly cleaved mica surface consists of negatively charged surface groups $0.52 \mathrm{~nm}$ apart in a nearly-hexagonal (tetragonal) lattice, about half of which are neutralised by potassium ions. When EAN is contacted with mica, the ethylammonium ions will adsorb to available surface charge sites, and compete with potassium for the occupied charged sites. Because (1) the concentration of ethylammonium in the AFM cell must be much greater than that of potassium, (2) the charged on ethylammonium is hard and localised, and (3) ethylammonium is known to be surface active, ${ }^{41}$ it is reasonable to assume that potassium is effectively expelled from all surface sites. Images obtained at high force support this supposition. Open to question is how the hexagonal lattice of mica and the bulk sponge morphology of EAN combine to produce the worm structures present in the image.

EAN's bulk nanostructure is due to solvophobic and van der Waals attractions between alkyl groups and it is these forces that also produce the observed surface structures. Consider ethylammonium ions adsorbed to surface charged sites, which for mica are $0.52 \mathrm{~nm}$ apart. The cross-sectional dimension of ethylammonium in this high ionic strength environment will be determined by the physical size of the alkyl chain ${ }^{3}$ so another ethylammonium ion can pack between the surface adsorbed cations due to solvophobic attractions with an associated nitrate to satisfy its charge. The inclusion of nitrate groups in the surface layer impedes alkyl chain aggregation in certain directions, because ethyl groups are too short to interact across the anions. ${ }^{42}$ The worm structures in the AFM images thus represents the ion morphology that maximises attractive interactions between cation alkyl chains and the electrostatic requirements of both the surface and the ions. Note that the potential of the surface layer need not be net neutral; several studies have suggested that the innermost layer overscreens the surface charge ${ }^{41}$ (in this case would be cation rich) but is balanced by the potential of subsequent transition zone layers. Attractions between alkyl groups could account for the concentration of cations in the innermost layer being greater than that required to neutralise the surface charge, analogous to charge reversal that occurs in cationic surfactant adsorption onto mica and mineral oxides. ${ }^{43}$

The near surface layer in Fig. 1(d) is less ordered than the innermost layer. Cations in this layer are solvophobically attracted to the (carbon chains of) surface-bound ions. Hence the effect of the mica surface on this layer is diminished, and the sponge morphology of the bulk liquid begins to reassert itself, resulting in a softer, more undulating, appearance. Attractions between this layer and the innermost layer hold it in place strongly enough to permit imaging in soft contact mode, but images of subsequent layers could not be obtained.

Fig. 2 shows the innermost EAN layer imaged at much higher force $(\sim 3 \mathrm{~V}$ over setpoint). This reveals the three lattice directions (indicated by the lines) of the mica substrate, but the appearance is markedly different to the bare surface. The lattice direction is most obvious in the direction across the image. The same orientation as the worms in previous soft contact scans of the same area of the surface (not shown). This is therefore the direction of the highest density of ions in the innermost layer. The spacing between rows varies between $0.48 \mathrm{~nm}$ for the most vertical axis to $0.58 \mathrm{~nm}$ for the most horizontal axis. As the AFM is most sensitive to horizontal periodicity, the spacing determined for the vertical axis is the most accurate, and this 


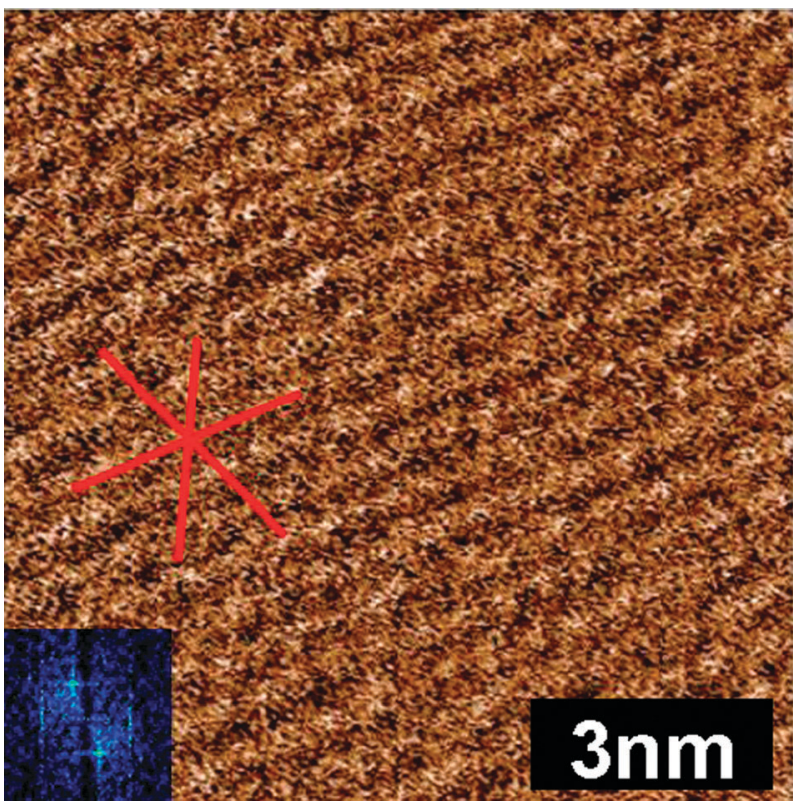

Fig. 2 Soft contact AFM deflection image of the EAN innermost layer on mica. The scan direction is down the image. The lines drawn on the image are to guide the eye, and show the three directions of the underlying mica lattice that templates ion adsorption.

value is consistent with the spacing between charges on the mica surface. At the high force used for this image, solvophobically associated ions (or ion pairs) in the innermost layer will be expelled by the AFM tip during imaging, and the cations that remain in place will have their alkyl groups flattened against the surface, such that the worm-like morphology is lost. In a recent article, we suggested that a change in the friction coefficient at high force for a similar system could be a consequence of just such a change in the innermost layer structure. ${ }^{34}$ Comparison of the image shown in Fig. 2 with Fig. 1(c) supports this, and it follows that the strongly flattened cation layer is more lubricating than the worm like structures.

Force-distance approach curves recorded for a mica surface immersed in EMIm TFSI ${ }^{6}$ were similar to those for EAN, with three clear steps on tip approach and an increasing force required to rupture layers closer to the surface. Wider steps of $0.75 \mathrm{~nm}$ are found between EMIm TFSI layers $(c f .0 .55 \mathrm{~nm}$ for EAN), consistent with a larger ion pair diameter (determined from the liquid density ${ }^{39}$ ). Soft contact images of the interfacial layers of EMIm TFSI are not sufficiently clear to reveal any lateral structure. At relatively low force the AFM tip displaces the innermost ion layer from the surface and comes into contact with the mica. This confirms that the highly localised ethylammonium ionic charge is more strongly bound to the substrate than is the delocalised imidazolium cation. We therefore used a gentler AM-AFM imaging mode with a range of cantilever stiffnesses to examine the innermost and transition zone layer structures in this system.

Amplitude- and phase-distance approach curves for the vibrating AFM tip moving towards the mica substrate immersed in EMIm TFSI are presented in Fig. 3. The oscillations in the

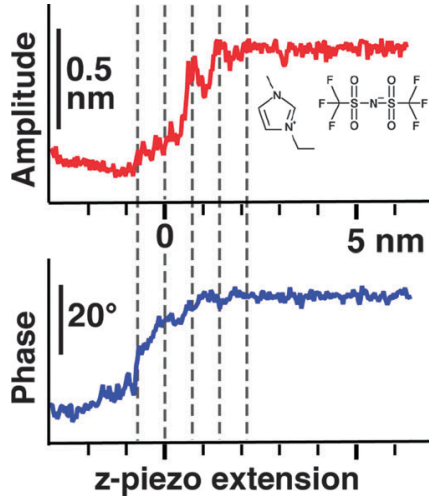

Fig. 3 Amplitude (top) and phase (bottom) data recorded as the vibrating AFM tip approaches the mica substrate immersed in EMIm TFSI. The $x$-axis measures the extension of the base of the cantilever and the zero is set arbitrarily. Distinct maxima are visible in the amplitude curve (dashed lines). The lines are $\sim 0.8 \mathrm{~nm}$ apart, a distance consistent with the ion pair dimension. Steps tend to be less clear in the phase data. The molecular structure of EMIm TFSI is shown as an inset to the amplitude figure.

amplitude data are a consequence of variations in the IL's molecular density profile normal to the interface. ${ }^{39}$ Similar oscillations have been observed for both protic and aprotic ILs confined between mica sheets using the highly sensitive surface forces apparatus, ${ }^{35,39}$ confirming that AM mode is more sensitive than contact mode AFM for probing IL interfacial structure. The distance between consecutive maxima is $0.8 \pm 0.2 \mathrm{~nm}$, which corresponds to the molecular dimensions of EMIm TFSI and the contact mode step size measured previously. ${ }^{6}$ The oscillations are less marked in the phase data, which decreases monotonically as the tip approaches the mica surface. The phase was therefore used to determine which interfacial region was scanned by AFM tip during AM imaging (see ESI $\dagger$ ).

A stiff cantilever $\left(k_{\mathrm{c}} \approx 6 \mathrm{~N} \mathrm{~m}^{-1}\right)$ was used to probe the IL structure nearest the surface. Images were obtained while the pressure exerted by the tip was progressively increased; the imaging setpoint is effectively decreased by lowering the ratio of the working amplitude over the free tip vibration amplitude $A / A_{0}$. This results in the average phase decreasing as the tip progressively removes ion layers until it reaches the innermost ion layer.

The final transition zone layer is presented in Fig. 4(a). The features in this image have mainly a hexagonal symmetry, indicated by the solid lines, but are $\sim 1.4 \mathrm{~nm}$ apart, which is more than double the mica lattice dimension. This suggests the mica lattice is influencing but not controlling the morphology of this layer. When the imaging setpoint is decreased, this layer is removed and images of the innermost layer are obtained, presented in Fig. 4(b) and at higher magnification in Fig. 4(c). These images reveal rows $\sim 0.5 \mathrm{~nm}$ apart (dashes lines in (c)) with the hexagonal symmetry of the mica lattice clearly visible (indicated by the solid lines). A height profile obtained along the line in Fig. 4(c) shows that the surface is much rougher than atomically flat mica. The phase contrast between points corresponding to maxima and minima in topography profile indicate that the AFM tip contacts the mica surface in the valleys, while 

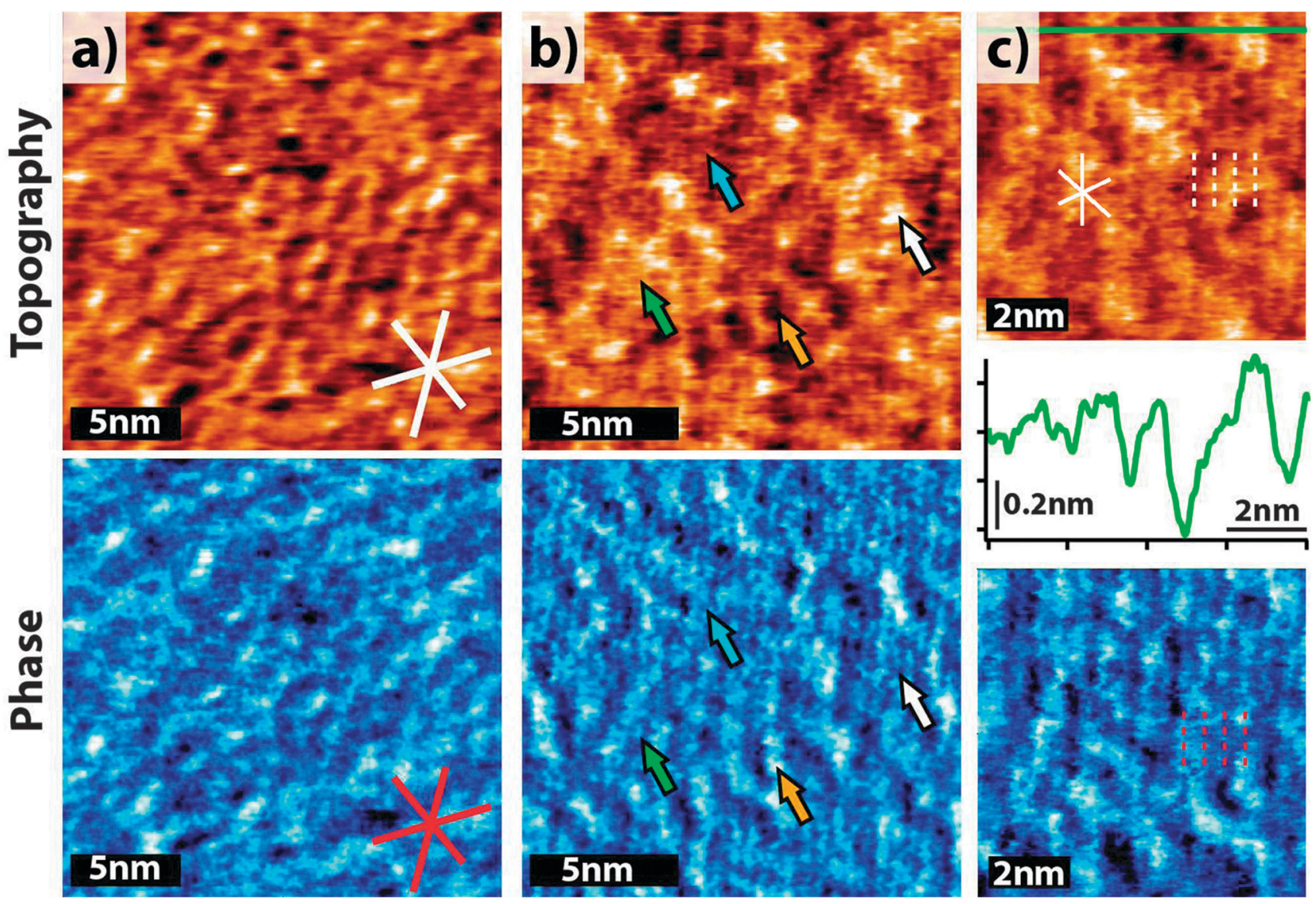

Fig. 4 Topographic and phase images of the innermost and first transition zone layer structure for the EMIm TFSI-mica system. (a) The first transition zone layer with the lines indicating $60^{\circ}$ symmetry. (b) Innermost layer. The arrows indicate areas of different heights, for explanation see text. (c) Innermost layer at higher magnification. The solid lines show the $60^{\circ}$ symmetry while the dashed vertical lines indicate rows separated by $0.5 \mathrm{~nm}$. The section analysis was taken across the green line in (c).

the peaks are associated with adsorbed ions, which suggests that the $\mathrm{EMIm}^{+}$layer adsorbed to the substrate is $\sim 0.4 \mathrm{~nm}$ thick. AFM does not reveal the orientation of the cation to the surface, but VSFS studies on solid surface have suggested the cation is adsorbed with the imidazolium ring slightly tilted towards the silica surface (between $16^{\circ}$ and $32^{\circ}$ from surface normal), tending to more parallel orientations with decreasing alkyl chain length, ${ }^{19,20}$ which is consistent with the layer thickness suggested by the height profile.

The imaging force used in Fig. 4(b) allows four features to be discerned. The mica substrate and innermost layer are indicated by the orange and blue arrows respectively. The first transition zone layer is the thickness of an ion pair, so can be divided into two sub-layers of approximately equal height. Both are present in Fig. 4(b), with the ion sub-layer closest to the surface indicated by the green arrow, and the sub-layer farther from the surface by the white arrow. These features were routinely observed in these imaging conditions.

Unlike EAN's worm-like morphology, the clear images of discrete ions in the EMIm TFSI innermost layer indicates that alkyl chain aggregation does not occur, which is consistent with the bulk structure. ${ }^{4}$ This suggests EMIm $^{+}$adsorbs onto a negative mica surface site, but in the absence of self assembly, anions are not appreciably incorporated into the surface layer, so the mica lattice controls adsorption. The next ion layer (i.e. the first transition zone layer) is of mixed composition, compensating the net potential of the mica and innermost layer.

Images of the transition zone structure are presented in Fig. 5. The sequence of images illustrates controlled removal of an ion layer with the scanning AFM tip $\left(k_{\mathrm{c}} \approx 0.76 \mathrm{~N} \mathrm{~m}^{-1}\right)$ in AM mode. The upper panels present topographic images and the lower panels the corresponding phase image, which were acquired simultaneously and provide an indication of the sample's viscoelasticity. In Fig. 5(a), stable imaging of an ionic layer is achieved, with molecular level details visible, and the phase image appears remarkably even. Combined, this suggests a cohesive layer with homogenous mechanical properties. From Fig. 5(b) to (d), the scanning tip presses incrementally harder, resulting in the progressive 'pealing' of an ion layer. Controlled layer removal is also visible in the phase images, with uncovered layers appearing darker; this phase decrease indicates that the layer closer to the mica is more robust.

To image the features of an ion layer at the edge of the transition zone a particularly soft cantilever $\left(k_{\mathrm{c}} \approx 0.09 \mathrm{~N} \mathrm{~m}^{-1}\right)$ was used. This lever was unable to remove ionic layers and move closer to the surface, but was able to reveal the structure 

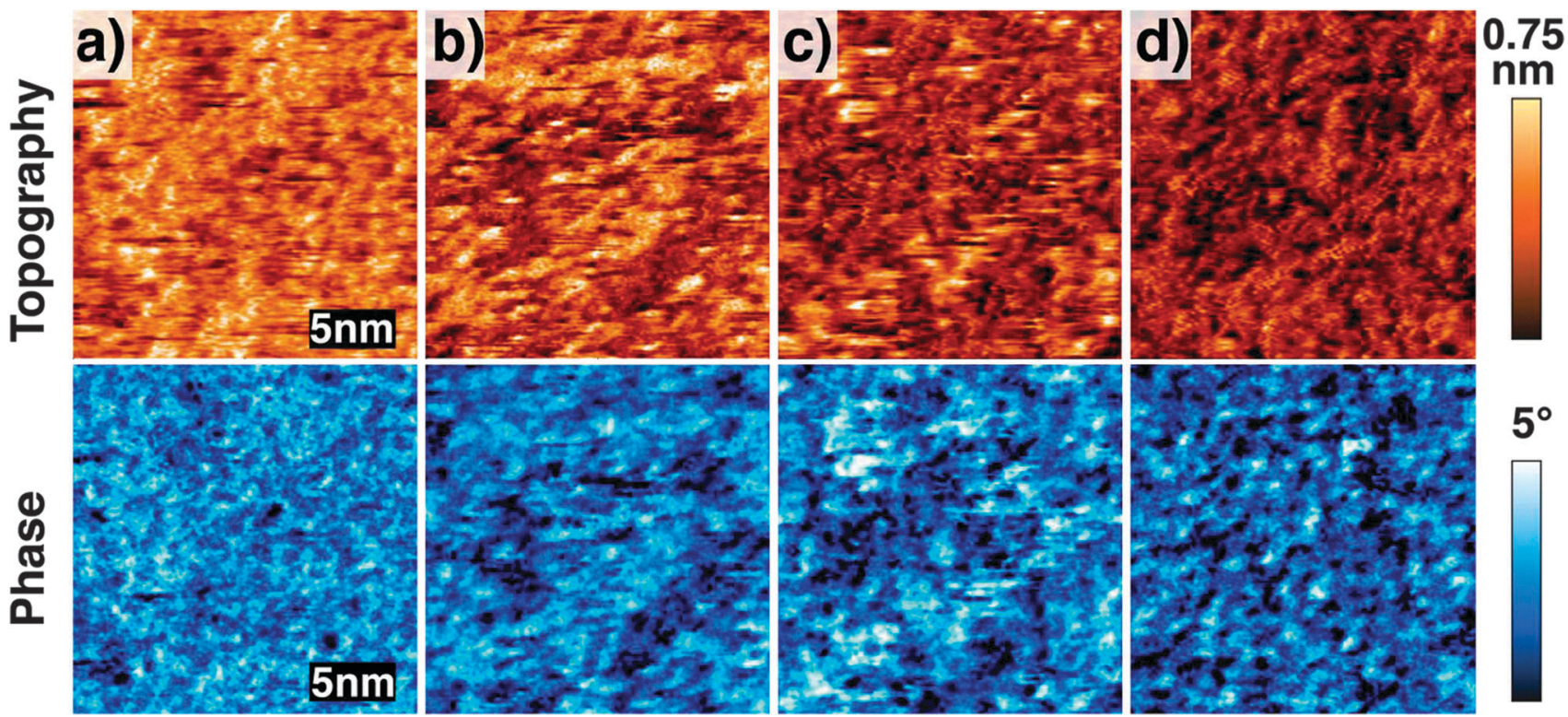

Fig. 5 Topographical and phase images of controlled removal EMIm TFSI transition zone layers are shown sequentially from a to d, which is achieved by progressively lowering the imaging setpoint.
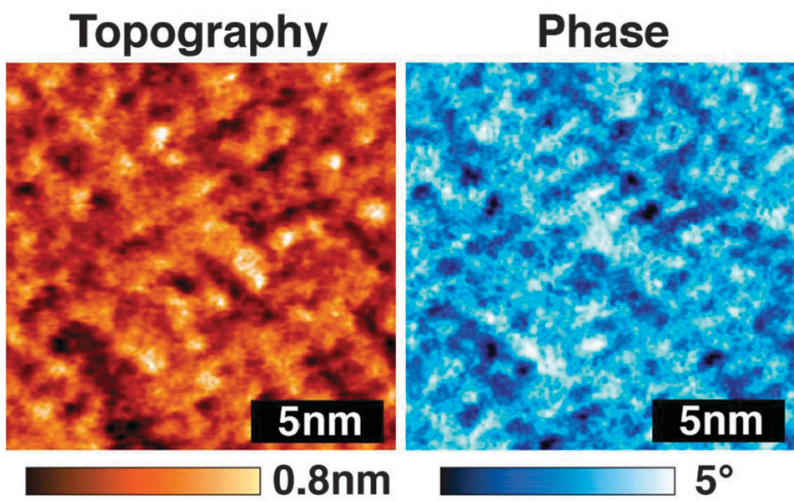

Fig. 6 Imaging of an outer EMIm TFSI transition zone layer using a particularly soft cantilever $\left(k_{c} \approx 0.09 \mathrm{~N} \mathrm{~m}^{-1}\right)$. $90^{\circ}$ symmetry is visible in both the phase and amplitude images. The typical distance between features is $\sim 1.3 \mathrm{~nm}$.

of an outmost transition zone layer. The lateral morphology consists of semi ordered rows dominated by a $90^{\circ}$ symmetry, cf. Fig. 6. This symmetry is markedly different to the $60^{\circ}$-dominated symmetry induced by the mica substrate closer to the surface, and indicates strong lateral cohesion within layers. $90^{\circ}$ symmetry could be induced by EMIm TFSI pairs arranging in a checkerboard fashion, which is broadly consistent with the bulk structure reported for EMIm TFSI. ${ }^{4}$

The transition from $90^{\circ}$ symmetry in the outmost transition zone layers to $60^{\circ}$ symmetry close to the mica surface occurs progressively. A movie of this sequence is included in the ESI $\dagger$ (movie S1). The phase data indicates the progression of the tip towards the substrate. At the beginning of the movie, the distance between the tip and the surface is large and outer transition zone layers with $90^{\circ}$ symmetry are imaged. As the tip moves towards the substrate the $60^{\circ}$ symmetry begins to appear and eventually dominates close to the surface. Interestingly, the structures in the images became more stable as the tip-surface separation distance decreased, which suggests that the mobility of ions within layers decreases. This result is consistent with high lateral cohesion.

In all the measurements presented here the IL is confined between the AFM tip and the mica surface, and effects from the measuring tip on the observed IL structure cannot be excluded; this is a general limitation common to all local probe techniques. However, the AFM tip is rougher and has a lower surface charge density than mica; both effects mean that surface interfacial structure will be more strongly a consequence of the mica substrate than the tip. ${ }^{12}$ We are confident that interfacial structure exists independently of the measuring process and are not imaging artifacts induced by the AFM tip for several reasons. Firstly, the features in the AFM images scale correctly with imaging size and rotate appropriately as the scan angle is changed. Secondly, the general features of the interface are consistent for both imaging modes even though the mechanism by which data is acquired is quite different. Thirdly, the change in the IL lateral organization through transition zone layers and the influence of the mica substrate are physically reasonable, and can be rationalized in terms of the accepted structures of the bulk liquid and mica substrate. ${ }^{44}$ Finally, the results obtained are consistent with molecular dynamic simulations ${ }^{45}$ and experimental results (e.g. sum frequency generation spectroscopy ${ }^{46,47}$ and X-ray reflectivity ${ }^{18}$ ) of similar ILs near solid interfaces.

\section{Conclusions}

In 1994, soft contact AFM images of self assembled surfactant aggregates adsorbed at solid surfaces from aqueous surfactant solutions $^{48,49}$ revolutionised our understanding of these 
systems. ${ }^{43}$ The structures elucidated here for EAN and EMIm TFSI will similarly impact solid-IL systems. While lateral structure is present in the innermost and transition zone layers for both liquids, there are important differences that can be correlated with differences in the ions' molecular structure and their ability to self assemble. Imaging reveals that ethylammonium remains adsorbed to the mica surface up to high force, whereas the EMIm cation is desorbed at relatively low pressures. This difference is attributed to the electrostatic attractions between surface charged groups and ethylammonium's localised charged group being stronger than those with the delocalized group of the EMIm ion. This suggests that for applications where strong surface adsorption is desirable, such as lubrication, charge localized ions should be employed, while for applications where access to the surface is required, such as for electrodeposition and dye solar cells, charge delocalized ions are preferable as solute adsorption from the solution will occur more readily. The fact that EAN self assembles both at the interface and in the bulk liquid and EMIm TFSI does not, suggests that EAN's hydrogen bond network provides additional cohesive interactions that promote self assembly of the ethyl chain, and that hydrogen bonding can be used as a tuning parameter for controlling IL nanostructure. In both ILs, the level of lateral cohesion within layers is high, elucidated by the uniformity of the phase images recorded for EMIm TFSI and the fact that soft contact images can be obtained for EAN. This finding has implications for fluid dynamics in $\mathrm{ILs}^{33}$ and the stability of particle suspensions. ${ }^{28,50}$

\section{Acknowledgements}

JJS and KV are grateful to Hauke A. Harms and Shaik M. Zakeeruddin for useful conversations and for kindly providing the EMIm TFSI. KV acknowledges funding from the Swiss National Science Foundation through the Ambizione Award and the Subsidy FNS 200021_140533 for support of projects. This research was supported by an Australian Research Council Discovery Project (DP120102708).

\section{References}

1 H. N. Patrick, G. G. Warr, S. Manne and I. A. Aksay, Langmuir, 1997, 13, 4349-4356.

2 R. Atkin and G. G. Warr, J. Phys. Chem. B, 2008, 112, 4164-4166.

3 R. Hayes, S. Imberti, G. G. Warr and R. Atkin, Phys. Chem. Chem. Phys., 2011, 13, 3237-3247.

4 M. Deetlefs, C. Hardacre, M. Nieuwenhuyzen, A. A. H. Padua, O. Sheppard and A. K. Soper, J. Phys. Chem. B, 2006, 110, 12055-12061.

5 T. L. Greaves, A. Weerawardena, C. Fong, I. Krodkiewska and C. J. Drummond, J. Phys. Chem. B, 2006, 110, 22479-22487.

6 R. Hayes, S. Z. El Abedin and R. Atkin, J. Phys. Chem. B, 2009, 113, 7049.

7 A. Ray, Nature, 1971, 231, 313-315.
8 C. A. Tanford, The Hydrophobic Effect: Formation of Micelles and Biological Membranes, Wiley, New York, 1980.

9 R. Hayes, S. Imberti, G. G. Warr and R. Atkin, Phys. Chem. Chem. Phys., 2011, 13, 13544-13551.

10 A. Triolo, O. Russina, H. J. Bleif and E. DiCola, J. Phys. Chem. $B, 2007,111,4641-4644$.

11 W. A. Hamilton, L. Porcar, P. D. Butler and G. G. Warr, J. Chem. Phys., 2002, 116, 8533-8546.

12 R. Atkin and G. G. Warr, J. Phys. Chem. C, 2007, 111, 5162-5168.

13 R. Hayes, G. G. Warr and R. Atkin, Phys. Chem. Chem. Phys., 2010, 12, 1709-1723.

14 R. Atkin, L. M. De Fina, U. Kiederling and G. G. Warr, J. Phys. Chem. C, 2009, 113, 12201.

15 R. Hayes, D. Wakeham and R. Atkin, in Ionic Liquids UNCOILed: Critical Expert Overviews, ed. N. Plechkova and K. Seddon, John Wiley and Sons, 2013.

16 R. Atkin, N. Borisenko, M. Druschler, S. Z. El Abedin, F. Endres, R. Hayes, B. Huber and B. Roling, Phys. Chem. Chem. Phys., 2011, 13, 6849-6857.

17 R. Hayes, N. Borisenko, M. K. Tam, P. C. Howlett, F. Endres and R. Atkin, J. Phys. Chem. C, 2011, 115, 6855-6863.

18 M. Mezger, H. Schroder, H. Reichert, S. Schramm, J. S. Okasinski, S. Schoder, V. Honkimaki, M. Deutsch, B. M. Ocko, J. Ralston, M. Rohwerder, M. Stratmann and H. Dosch, Science, 2008, 322, 424-428.

19 B. D. Fitchett and J. C. Conboy, J. Phys. Chem. B, 2004, 20255. 20 J. B. Rollins, B. D. Fitchett and J. C. Conboy, J. Phys. Chem. B, 2007, 111, 4990.

21 D. Wakeham, P. Niga, C. Ridings, G. Andersson, A. Nelson, G. G. Warr, S. Baldelli, M. W. Rutland and R. Atkin, Phys. Chem. Chem. Phys., 2012, 14, 5106-5114.

22 C. Ridings, G. G. Warr and G. G. Andersson, Phys. Chem. Chem. Phys., 2012, 14, 16088-16095.

23 A. A. Kornyshev, J. Phys. Chem. B, 2007, 111, 5545-5557.

24 K. B. Oldham, J. Electroanal. Chem., 2008, 613, 131.

25 Y. Lauw, M. D. Horne, T. Rodopoulos and F. A. M. Leermakers, Phys. Rev. Lett., 2009, 103, 117801.

26 J. Wu, T. Jiang, D.-e. Jiang, Z. Jin and D. Henderson, Soft Matter, 2011, 7, 11222-11231.

27 N. Jain, X. Zhang, B. S. Hawkett and G. G. Warr, ACS Appl. Mater. Interfaces, 2011, 622-667.

28 J. A. Smith, O. Werzer, G. B. Webber, G. G. Warr and R. Atkin, J. Phys. Chem. Lett., 2010, 1(1), 64-68.

29 D. R. MacFarlane, J. M. Pringle, P. C. Howlett and M. Forsyth, Phys. Chem. Chem. Phys., 2010, 12, 1659-1669.

30 M. Armand, F. Endres, D. R. MacFarlane, H. Ohno and B. Scrosati, Nat. Mater., 2009, 8, 621-629.

31 F. Endres, ChemPhysChem, 2002, 3, 144-154.

32 M. Gorlov and L. Kloo, Dalton Trans., 2008, 2655-2666.

33 R. Asencio, E. D. Cranston, R. Atkin and M. W. Rutland, Langmuir, 2012, 28, 9967-9976.

34 O. Werzer, E. D. Cranston, G. G. Warr, R. Atkin and M. W. Rutland, Phys. Chem. Chem. Phys., 2012, 14, 5147-5452.

35 S. Perkin, T. Albrecht and J. Klein, Phys. Chem. Chem. Phys., 2010, 12, 1243-1247. 
36 K. Voitchovsky, J. J. Kuna, S. A. Contera, E. Tosatti and F. Stellacci, Nat. Nanotechnol., 2010, 5, 401-405.

37 R. Hayes, G. Warr and R. Atkin, Phys. Chem. Chem. Phys., 2010, 12, 1709-1723.

38 D. Wakeham, R. Hayes, G. G. Warr and R. Atkin, J. Phys. Chem. B, 2009, 113, 5961-5966.

39 R. G. Horn, D. F. Evans and B. W. Ninham, J. Phys. Chem., 1988, 92, 3531.

40 D. Choy and S.-H. Chen, Phys. Rev. E: Stat. Phys., Plasmas, Fluids, Relat. Interdiscip. Top., 2000, 61, 4148.

41 D. Wakeham, D. Eschebach, G. B. Webber, R. Atkin and G. G. Warr, Aust. J. Chem., 2012, 65, 1554-1556.

42 T. Iwahashi, T. Nishi, H. Yamane, T. Miyamae, K. Kanai, K. Seki, D. Kim and Y. Ouchi, J. Phys. Chem. C, 2009, 113, 19237.
43 R. Atkin, V. S. J. Craig, E. J. Wanless and S. Biggs, Adv. Colloid Interface Sci., 2003, 103, 219-304.

44 O. Werzer, E. Cransten, G. G. Warr, R. Atkin and M. R. Rutland, Phys. Chem. Chem. Phys., 2012, 14, 5147.

45 K. Shimizu, A. Pensado, P. Malfreyt, A. A. H. Padua and J. N. Canongia Lopes, Faraday Discuss., 154, 155-169.

46 B. D. Fitchett and J. C. Conboy, J. Phys. Chem. B, 2004, 108, 20255-20262.

47 J. B. Rollins, B. D. Fitchett and J. C. Conboy, J. Phys. Chem. B, 2007, 111, 4990-4999.

48 S. Manne, J. P. Cleveland, B. E. Gaub, G. D. Stucky and P. K. Hansma, Langmuir, 1994, 10, 4409-4413.

49 S. Manne and H. E. Gaub, Science, 1995, 270, 1480-1482.

50 K. Ueno, S. Imaizumi, K. Hata and M. Watanabe, Langmuir, 2009, 25, 825. 